\title{
Vehicle crash test against a lighting pole: experimental analysis and numerical simulation
}

\author{
G. Janszen \\ Aerospace Engineering Department, Politecnico di Milano, Italy
}

\begin{abstract}
The role of safety on the road is getting more and more important due to the overall increase of traffic. Active and passive safety deals with different concepts, the first to prevent an accident, the second to reduce the risks of occupant injury during an accident, but both lead to the same objective: safety. In this work, the role of a crashworthy lighting pole is discussed in order to assure a higher level of safety. Crash tests on an existing aluminium alloy lighting pole have been carried out, at the LAST Laboratory of the Aerospace Engineering Department of the Politecnico di Milano, to get sufficient data to create a numerical model of both the car and the lighting pole. Modifications to the pole have been investigated with the use of a multi-body program called VeDyAC, to verify the possibility of successfully fulfilling the European standard EN12767 "Passive safety of support structures for road equipment - Requirements and test methods". First results appear encouraging but further analysis has to be carried out, particularly on the base of the pole, which is the major part involved in the contact with the vehicle during the crash event. Results of experimental tests and numerical simulations with different solutions are presented and discussed.

Keywords: passive safety, crash tests, injury criteria, multibody analysis.
\end{abstract}

\section{Introduction}

The behaviour of an existing lighting pole (fig. 1) in a car crash event has been investigated in order to assess the crashworthiness of the pole itself. The work has been divided in three main areas, and the paper follows the same criteria. One area concerns the mechanical characterisation of the materials used for the pole. Another area covers the numerical simulation of the impact of a car with 
the existing pole and with two possible modified versions of it. The simulations were carried out with a home made multibody program that includes the creation of a numerical model of the pole and of a small car used in the dynamic tests. The last main area is related to the crash tests on the existing lighting pole and on its modifications, with a car and a rigid sled.

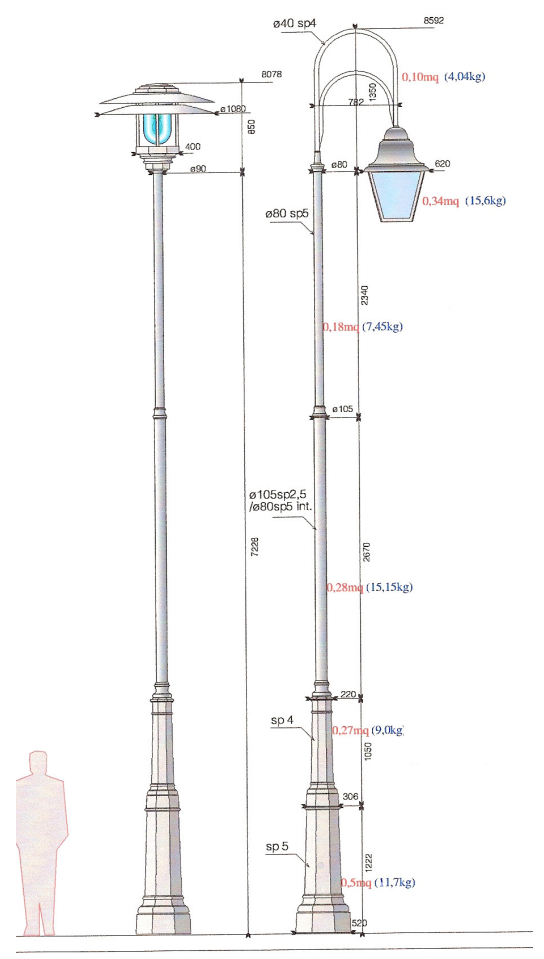

Figure 1: The existing lighting pole.

\section{Material characterization}

The lighting pole is made in three main parts: a casting aluminium alloy base divided in two parts with an octagonal section, a wrought aluminium alloy extruded tube with circular section also made in two parts, and finally the lantern and its support made of a casting aluminium alloy. Tensile tests have been carried out on specimens taken out from the base and the upper tube to get data for the numerical models. The tests have been performed using an MTS tensile test machine. The loads and the displacements were measured with a $250[\mathrm{kN}]$ full scale MTS load cell mod 661.22D-01 and an LVDT MTS transducer mod. 318.25 respectively. Figures 2 and 3 show the material stress-strain curves of the base and upper part respectively. Tables 1 and 2 show the mechanical characteristics of the materials tested. 


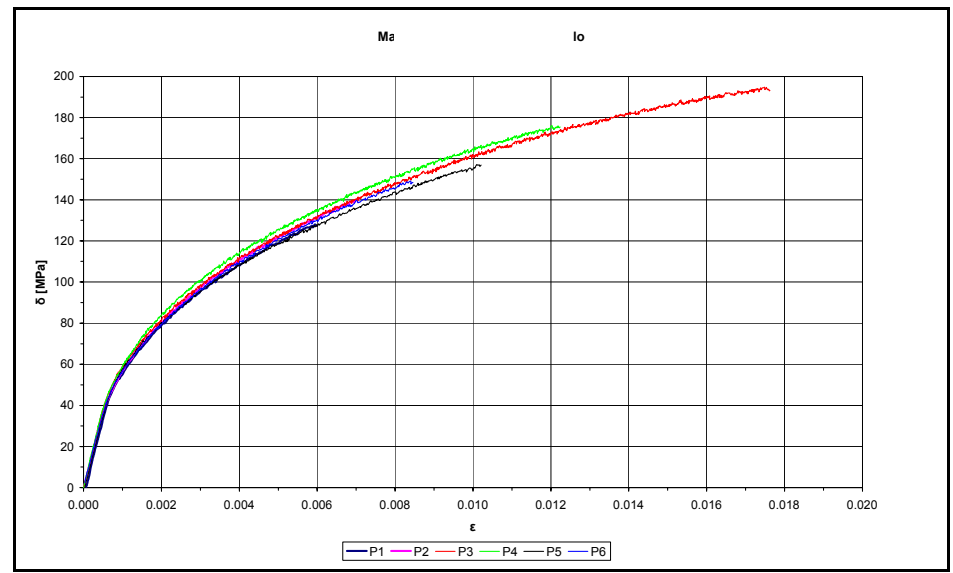

Figure 2: $\quad$ Stress-strain curves of the base's material.

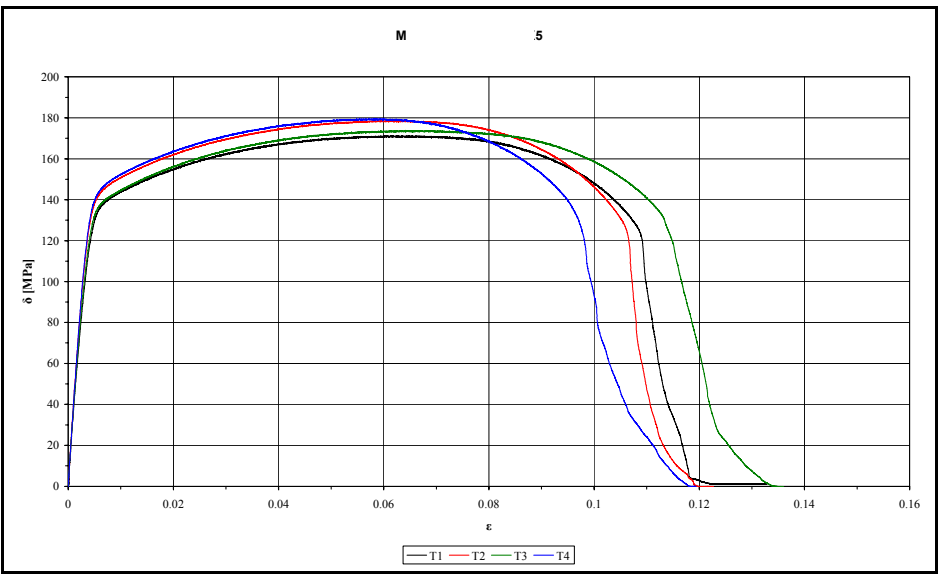

Figure 3: $\quad$ Stress-strain curves of the upper part's material.

Table 1: Mechanical characteristics of the base's material.

\begin{tabular}{|c|c|c|c|c|}
\hline Spec. & $\begin{array}{c}\text { Modulus of elasticity } \\
{[\mathrm{Mpa}]}\end{array}$ & $\begin{array}{c}\text { Yield stress } \\
{[\mathrm{Mpa}]}\end{array}$ & $\begin{array}{c}\text { Ultimate stress } \\
{[\mathrm{Mpa}]}\end{array}$ & $\begin{array}{c}\text { Max elongation } \\
{[\%]}\end{array}$ \\
\hline P1 & 75708 & 100 & 128 & 0.43 \\
\hline P2 & 73534 & 102.5 & 140 & 0.51 \\
\hline P3 & 78059 & 103 & 195 & 1.5 \\
\hline P4 & 77503 & 107 & 176 & 0.97 \\
\hline P5 & 74526 & 100 & 157 & 0.82 \\
\hline P6 & 71041 & 101.5 & 149 & 0.65 \\
\hline
\end{tabular}


Looking at the tables it can be noticed that the upper part exhibits a moderately large plastic behaviour if compared to that of the base, so that a three point bending test has been carried on it to investigate its capability to absorb impact energy.

Table 2: $\quad$ Mechanical characteristics of the upper part's material.

\begin{tabular}{|c|c|c|c|c|}
\hline Spec. & $\begin{array}{c}\text { Modulus of } \\
\text { elasticity } \\
{[\mathrm{Mpa}]}\end{array}$ & $\begin{array}{c}\text { Yield } \\
\text { stress } \\
{[\mathrm{Mpa}]}\end{array}$ & $\begin{array}{c}\text { Ultimate } \\
\text { stress } \\
{[\mathrm{Mpa}]}\end{array}$ & $\begin{array}{c}\text { Max } \\
\text { elongation } \\
{[\%]}\end{array}$ \\
\hline T1 & & 135 & 171 & 11 \\
\hline T2 & & 144 & 179 & 11.5 \\
\hline T3 & & 137 & 174 & 12.5 \\
\hline T4 & 69759 & 143 & 179 & 11 \\
\hline
\end{tabular}
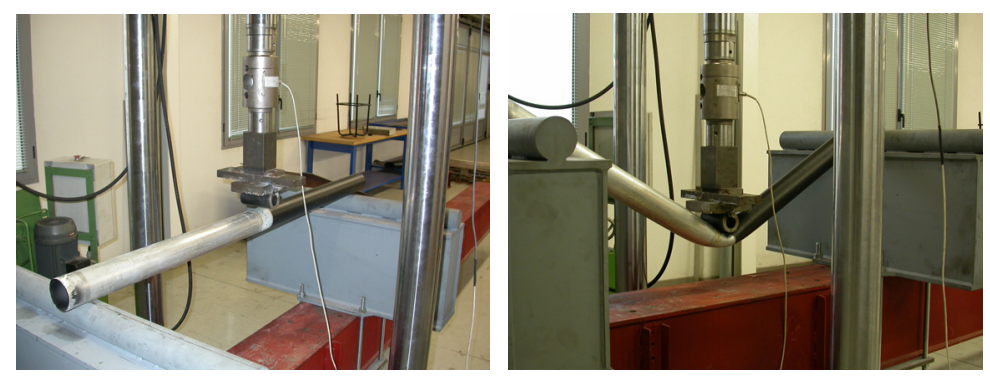

Figure 4: Three point bending test.

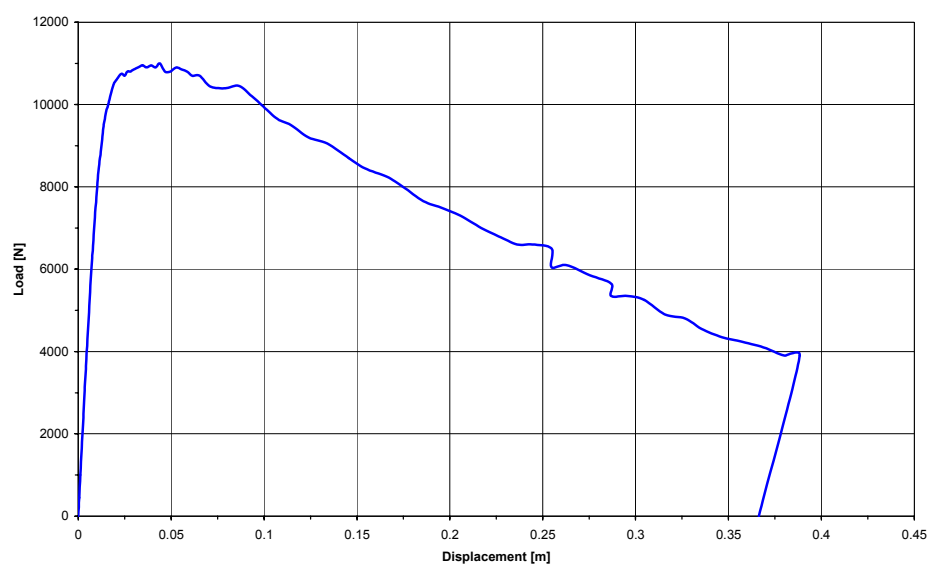

Figure 5: Bending load curve. 
In the test the distance between the external supports was set to 1420 [mm] and the load applied in the middle by means of a 5000 [daN] press. Load and displacements were measured and registered on file during the test. The yielding moment has been subsequently used in the numerical analysis to simulate the plastic hinge between the base and the upper part of the lighting pole.

The upper part has been further characterised by a dynamic test. The test has been carried out using a drop tower. This is capable of dropping an up to 1000 [kg] ballasted sled with a final velocity of about $10.5 \mathrm{~m} / \mathrm{s}$. The sled is instrumented with a 200 [g] ENTRAN EGCS-200 dumped piezoresistive accelerometer and a Tekel TK 462 encoder to measure the displacement over time. This facility only allows crash testing on small components, and has in fact been used to test only a section of the upper part. The direction of the impact was perpendicular to the side surface of the upper cylindrical part. The drop height was in this case $0.6[\mathrm{~m}]$ and the mass $53.2[\mathrm{~kg}]$; the final velocity was therefore $3.6[\mathrm{~m} / \mathrm{s}]$. Figure 6 shows the result of the test.

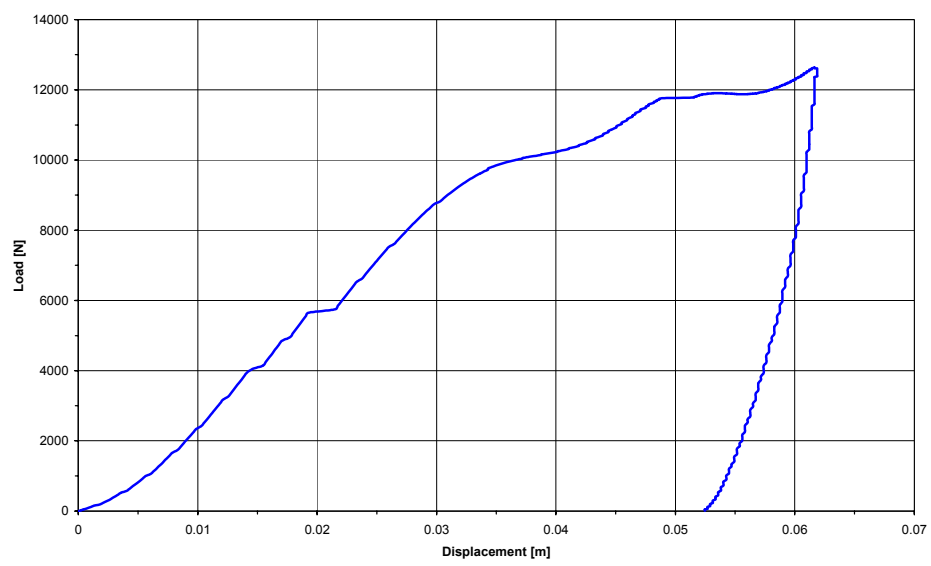

Figure 6: Dynamic compression load curve.

\section{Numerical analysis}

The numerical analysis have been carried out with a multibody code called VeDyAC [1] (Vehicle Dynamics And Crash) that was developed by the Aerospace Engineering Department of the Politecnico di Milano in co-operation with the Dutch Institution SWOV (Institute for Road Safety Research) in the 70 's. It is based on lumped parameter modelling and belongs to that category of codes, also referred as hybrid codes, in which the input data, such as deformable elements stiffness and plastic limits or contact surfaces characteristics, should be obtained mainly by experimental testing on sub-components of the entire structure, or even by manual computations if the sub-component is simple, or by finite element methods. The main components of a VeDyAC model are: rigid bodies, nodes, deformable elements and contact surfaces. 

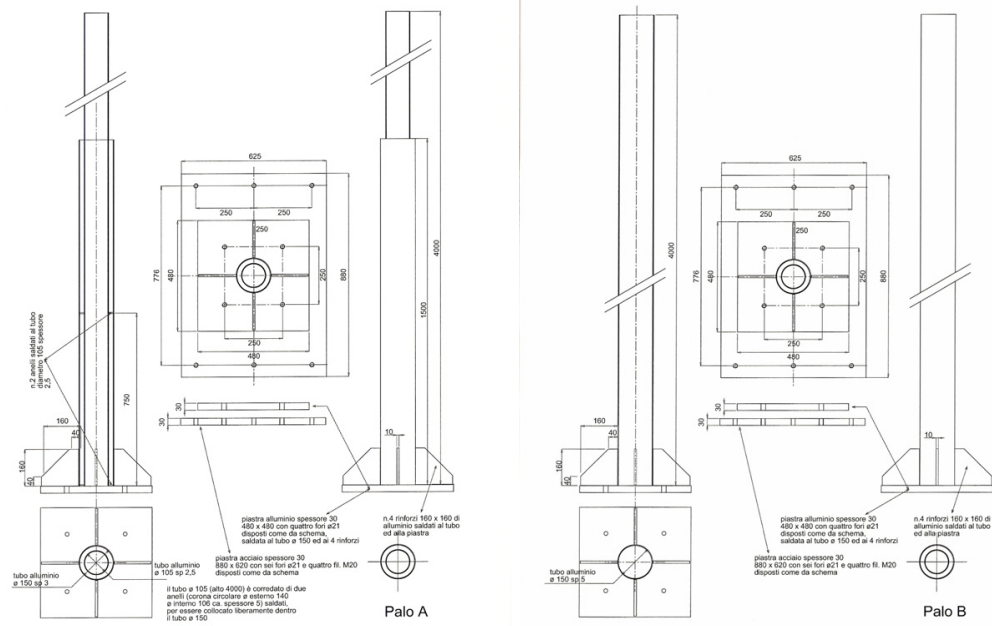

Figure 7: Modifications to the existing lighting pole.

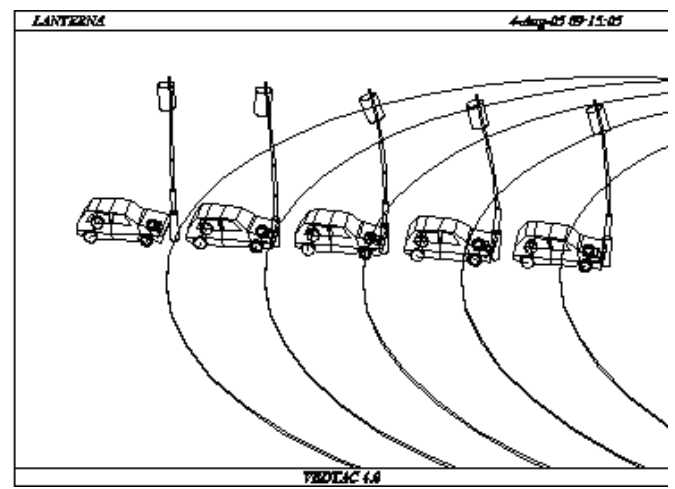

Figure 8: Numerical simulation of the impact against the existing lighting pole.

To simulate the impact of the car against the lighting pole this has been modelled with the following rigid bodies: 1) one for the lower part of the base which is linked to the ground; 2) one for the upper part of the base which is in connection with the upper cylindrical part; 3) two for the first part of the upper cylindrical element; 4) two for the second part of the upper cylindrical element; 5) one for the lantern and its support. The geometric and inertial characteristics have been extracted from the technical drawings. The different elements have been joined together with deformable elements to reproduce the stiffness of the pole. The characteristics of the material used for the different parts of the pole have been taken from the static and dynamic tests discussed in the previous chapter. The car reproduced the Italian FIAT UNO of $900[\mathrm{~kg}]$ mass also used in the crash tests. The impact speed was $50[\mathrm{~km} / \mathrm{h}]$. In figure 8 the numerical 
simulation of the impact against the existing pole is shown. It can be noticed how the pole does not exhibit plastic bending but only a shear-tear mode rupture. Figure 9 shows the simulation against a pole in which the base has been replaced by a cylindrical element made of the same material of the upper part of the existing pole with varying diameter and thickness, fig 7 . The bending phase before rupture at the base of the pole can be noticed in comparison with the behaviour of the brittle type of failure of the existing pole.

\section{Crash tests}

Crash tests have been performed at the LAST Laboratory of the Aerospace Engineering Department of the Politecnico di Milano [2]. Actually the lighting pole has been reduced in dimension from $8[\mathrm{~m}]$ to $6[\mathrm{~m}]$ to allow the test to be carried out inside the laboratory. The car is accelerated by means of a pneumatic system and a few meters before the impact area, the car is released from the hauling sledge with a constant speed. This is measured by a couple of Tag Heur photocells positioned at a fixed distance. High speed video cameras are installed close to the impact area to shoot the event. Three mutually perpendicular $50[\mathrm{~g}]$ ENTRAN EGCS-200 dumped piezoresistive accelerometers are installed at the CG of the car. The values of the accelerations have been used to evaluate the injury criteria [3]. Figures 10 and 11 show the installation of the test and the results.

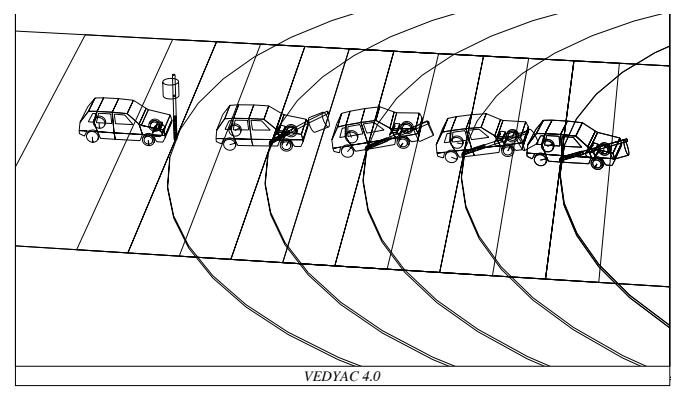

Figure 9: Numerical simulation of the impact against the modified lighting pole.
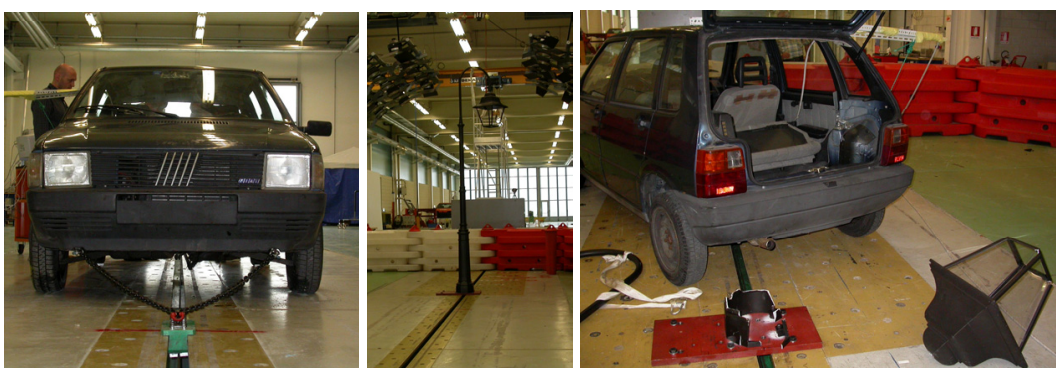

Figure 10: The car, the pole and the result of the test. 


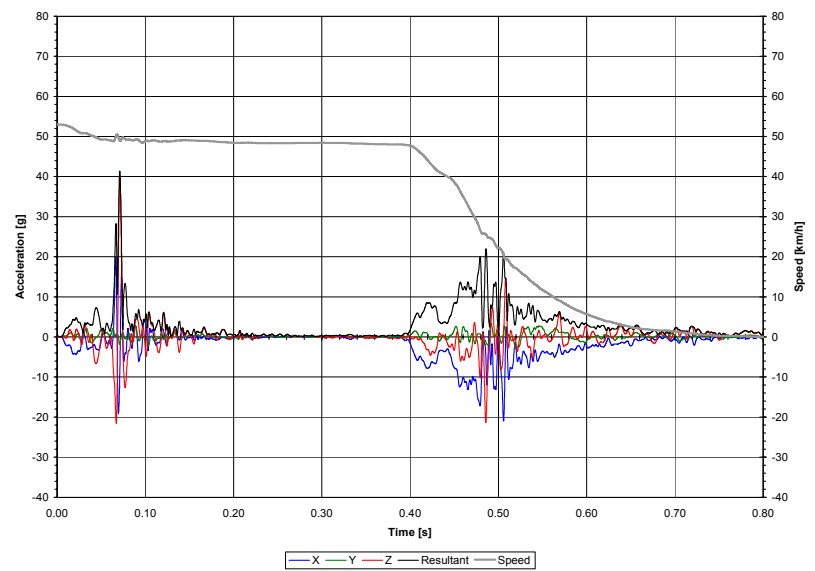

Figure 11: Acceleration and speed curves measured at the CG of the car.
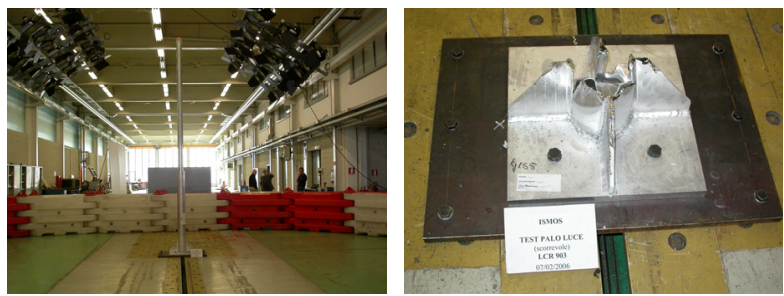

Figure 12: The first solution of the modified pole and the result of the test.

As can be noticed from the test, a brittle fracture occurs when a casting alloy is used in elements that are subjected to impacts. In order to avoid this kind of rupture, which must not be permitted causing possible injury to the occupants present in the impact area, a change in material has been made. Two more tests have been carried out on a modified pole made of the same material of the upper part of the existing pole (see fig 7). Figures 12-17 show the tests and their results.

\section{Conclusions}

From the results of the simulations and of the crash tests, it has been noticed that the improvement of the crashworthiness of the pole could be obtained not only with a change in the material used, which must necessarily have a wide plastic range and no brittle type of rupture, but with a new design of the base of the lighting pole. The interaction of the car and the pole is localized in this part and the loads derived from the impact are then transmitted to the ground. In this area a great variation of the stiffness between the base and the ground is concentrated, leading to the kind of rupture observed during the tests. Therefore the idea is to optimize the design of the base itself with different solutions represented in the following figures to make this stiffness variation as smooth as possible $[4,5]$. 
This will be done with the use of a finite element model of the base that will be subjected to the loads derived from the numerical and experimental analysis of the present work. With the aid of a home made optimization program the best solution between those illustrated in figure 18 will be investigated.
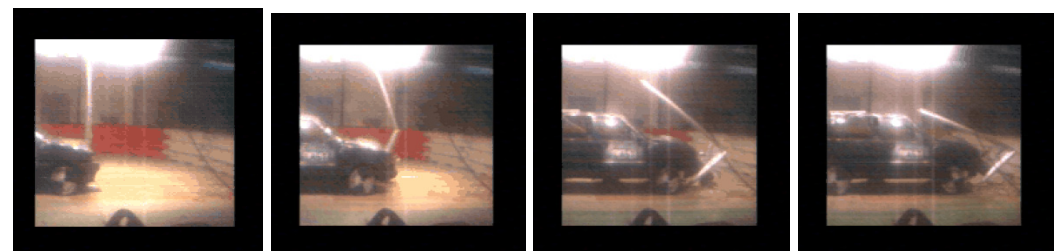

Figure 13: Frames from the high speed camera.

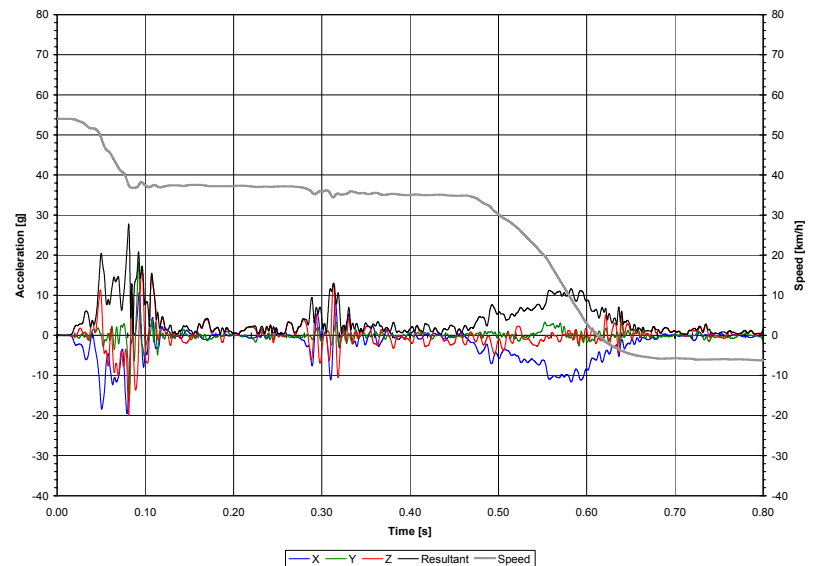

Figure 14: Acceleration and velocity curves measured at the $\mathrm{CG}$ of the car.
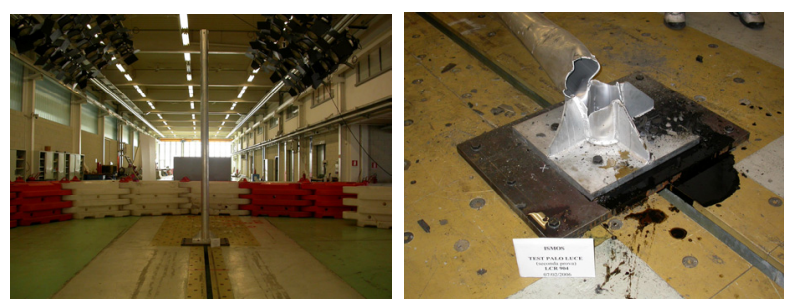

Figure 15: The second solution of the modified pole and the result of the test.

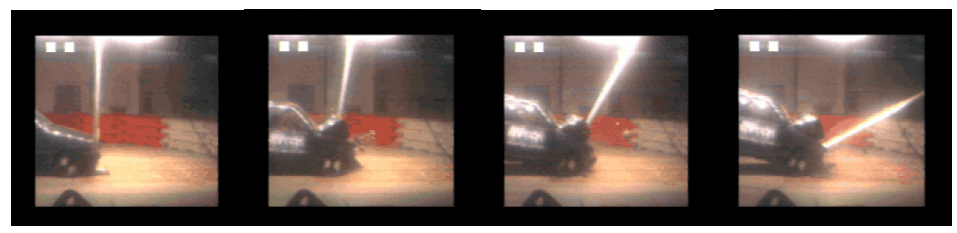

Figure 16: Frames from the high speed camera. 


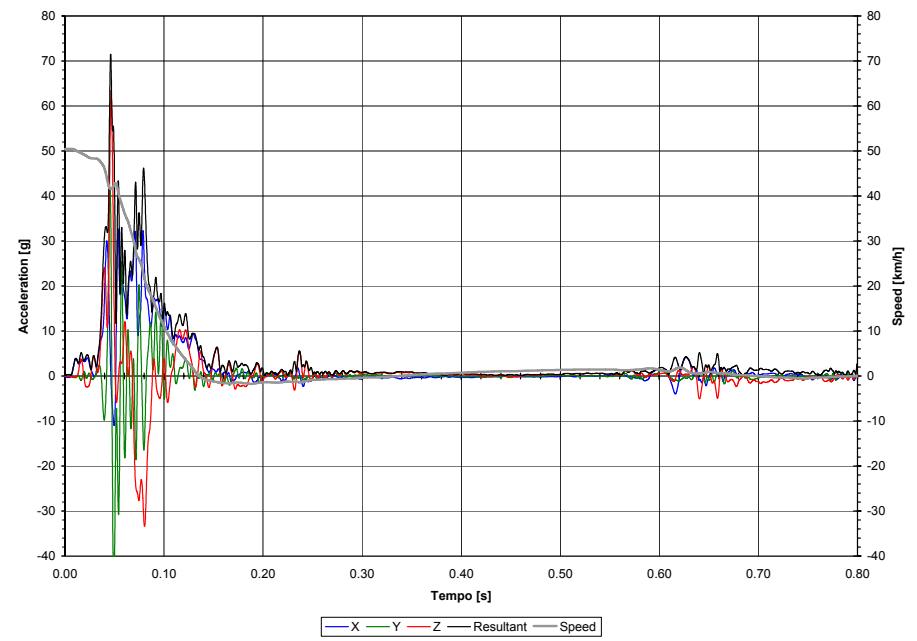

Figure 17: Acceleration and velocity curves measured at the CG of the car.
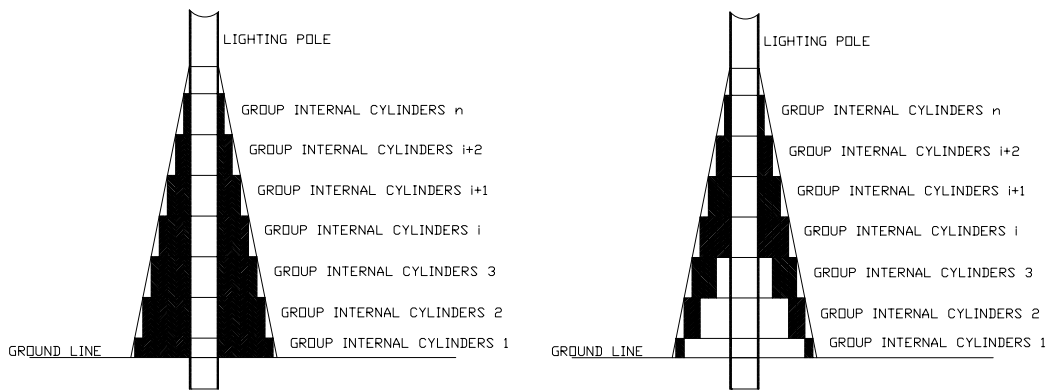

Figure 18: Two possible solutions.

\section{References}

[1] Giavotto, V., Puccinelli, L., Borri, M., Edelman, A., Heijer, T., Vehicle Dynamics and Crash Dynamics with Minicomputer. Computer and Structures, Volume 16, 1983.

[2] Dynamic Evaluation of Seat Restraint System \& Occupant Protection on Transport Airplanes, $A C$ 25.562-1, Federal Aviation Administration, 1989.

[3] Injury Criteria For Human Exposure to Impact. $A C$ No 21-22, Federal Aviation Administration, 1985.

[4] Singley, G. T., Haley, J. L., The Use of Mathematical Modeling in Crashworthy Seating Systems. AGARD CP-253, 1978

[5] Goldberg, D. E., Genetic algorithms in search, optimization, and machine learning. Addison Wesley, Reading, MA, 1989. 